Vol. 8, n 1 | 2004

Varia

\title{
Les origines de la statistique judiciaire sous la Révolution
}

\section{Emmanuel Berger}

\section{(2) OpenEdition \\ 12 Journals}

Édition électronique

URL : https://journals.openedition.org/chs/517

DOI : $10.4000 /$ chs. 517

ISSN : 1663-4837

Éditeur

Librairie Droz

Édition imprimée

Date de publication : 1 août 2004

Pagination : 65-91

ISBN : 2-600-00955-8

ISSN : 1422-0857

Référence électronique

Emmanuel Berger, «Les origines de la statistique judiciaire sous la Révolution », Crime, Histoire \& Sociétés / Crime, History \& Societies [En ligne], Vol. 8, $n^{\circ} 1$ | 2004, mis en ligne le 26 février 2009, consulté le 07 décembre 2022. URL : http://journals.openedition.org/chs/517 ; DOI : https://doi.org/ $10.4000 /$ chs. 517

Ce document a été généré automatiquement le 23 mars 2022.

Tous droits réservés 


\title{
Les origines de la statistique judiciaire sous la Révolution
}

\author{
Emmanuel Berger
}

\section{Introduction}

1 L'histoire de la statistique judiciaire a fait l'objet de nombreuses études au cours des dernières décennies ${ }^{2}$. Si les grandes lignes de cette histoire sont aujourd'hui relativement bien connues, force est de constater que l'étude de la période révolutionnaire a été quelque peu délaissée. Ce désintérêt semble avoir deux origines. La première est d'ordre historiographique. L'empreinte laissée par les recherches menées sur la statistique régionale et napoléonienne a modelé durablement l'image de l'ensemble de la statistique révolutionnaire. Celle-ci est, en partie, présentée de manière positive à travers la multiplicité des initiatives statistiques ${ }^{3}$, la collaboration enthousiaste entre savants et administrateurs et la formation d'un personnel compétent pour les régimes postérieurs. Mais, si la Révolution avait rassemblé les idées, les hommes et trouvé un projet ${ }^{4}$, elle ne réussit pas à réaliser ses différentes ambitions statistiques. Les principales causes de cet échec sont attribuées à l'instabilité du régime $^{5}$, à l'inadéquation entre l'ampleur des objectifs et l'insuffisance des moyens techniques et humains ${ }^{6}$ affectés à leur réalisation et enfin à l'absence d'un "réflexe statistique » parmi les fonctionnaires locaux ${ }^{7}$. Le Directoire échappe quelque peu à ce bilan critique puisqu'il est rattaché au Consulat, en tant qu'antichambre de l'âge d'or de la statistique régionale française ${ }^{8}$. L'image d'une statistique révolutionnaire généreuse mais incapable d'accoucher de résultats a pu contribuer à détourner les historiens de l'analyse de son versant judiciaire. Une telle image induit une absence de sources archivistiques suffisamment intéressantes pour y consacrer une étude. Le doute émis quant à la conservation des fonds d'archives constitue dès lors une seconde explication du désintérêt exprimé à l'encontre de la place occupée par la Révolution dans le développement de la statistique judiciaire. Il est d'ailleurs symptomatique que les premières contributions abordant cette thématique, au début des années 1990, consacrent une place centrale au traitement des archives. La première étude revient à 
Isabelle Guégan qui réalisa, à partir de dépouillements effectués aux Archives nationales, un inventaire des enquêtes administratives et des statistiques produites entre 1789 et $1795^{9}$. Son travail mit en évidence l'existence de plusieurs "enquêtes judiciaires ", jusque-là inconnues, et servit de base à l'étude que Xavier Rousseaux, Fred Stevens et Axel Tixhon entreprirent sur les origines de la statistique pénale en Belgique entre 1795 et $1835^{10}$. En s'appuyant sur un important travail de dépouillements archivistiques, les trois chercheurs réussirent à poser les jalons de la statistique pénale. Il est donc frappant d'observer que les principaux apports de ces recherches tiennent essentiellement à un travail archivistique de fond, qui n'avait jusqu'à présent jamais été réalisé.

2 Notre étude se situe dans la continuité de cette démarche. À partir d'un dépouillement systématique et parfois fastidieux des archives, nous avons voulu combler un vide et éclairer la contribution que la Révolution apporta à la construction de la statistique judiciaire. L'enjeu s'avère d'importance car, comme le résume avec un peu d'emphase Isabelle Guégan, il s'agit d'accorder aux trois premières assemblées nationales une place honorable dans l'histoire des progrès de la statistique ou au contraire de les renvoyer au néant dans lequel - faute de sources - l'historiographie les a laissées ${ }^{11}$. Parmi les différentes statistiques judiciaires lancées sous la Révolution, nous nous sommes limités à l'étude des "statistiques régulières $»^{12}$, produites pour une durée indéterminée et dont le but était d'obtenir des informations périodiques ${ }^{13}$. Ce choix a été dicté par l'état de conservation des archives et la nécessité de travailler sur des documents témoignant de résultats statistiques. La découverte de telles sources n'est pas aisée. Il n'existe, en effet, aucun fonds dénommé "statistiques judiciaires » et la majorité des archives ont disparu ou ont été dispersées. Pour se repérer dans ce dédale, il convient de suivre les différents "producteurs" d'archives de statistiques judiciaires que sont, au niveau central, les comités des assemblées législatives et le ministère de la Justice, au niveau départemental, les tribunaux criminels et, au niveau de l'arrondissement, les tribunaux correctionnels.

3 Avant d'aborder les résultats de notre recherche, il convient de faire deux remarques. La première est d'ordre " généalogique ». La statistique judiciaire n'est pas une création de la Révolution. Elle fait l'objet de plusieurs expériences sous l'Ancien Régime. L'ordonnance de 1670 demande aux procureurs du roi un relevé semestriel des crimes et délits. Les chanceliers d'Aguesseau et Lamoignon réitèrent ces demandes, en 1733 et 1760. D'autres ministres feront de même, par après, sans que leurs exigences soient suivies d'effet ${ }^{14}$. À la fin de l'Ancien Régime, les premiers résultats statistiques apparaissent à travers l'expérience de Montyon. Ce magistrat fait relever, dans les minutes du Parlement de Paris, le nombre et les caractéristiques sociologiques des condamnés criminels entre 1775 et 1786 et formule, à partir des données recueillies, des Observations sur la moralité en France. L'année suivante, en 1787, les Parlements sont contraints d'envoyer périodiquement des comptes rendus et des états des procès en matière criminelle mais les circonstances font avorter le projet $^{15}$. À la veille de la Révolution, les expériences semblent donc s'être multipliées, sans avoir réussi à produire des statistiques judiciaires régulières.

4 La seconde remarque touche à l'utilisation de la notion de statistique judiciaire. Il est nécessaire d'en définir clairement l'objet afin d'éviter toute ambiguïté sémantique. La statistique judiciaire est définie, aujourd'hui, comme un ensemble de données numériques concernant les institutions judiciaires et leur fonctionnement ${ }^{16}$ et l'on pourrait préciser 
qu'elles sont destinées à renseigner et aider les gouvernements ${ }^{17}$. Elle regroupe donc des informations nécessairement mises sous forme numérique concernant des domaines aussi diversifiés que la Justice pénale, la Justice civile, les crimes ou encore les prisons. Pendant la Révolution, le terme "statistique judiciaire» n'existe pas. On parle de comptes et, sous le Directoire, d'états. Toute la difficulté consiste à déterminer si les comptes ou états relèvent déjà de la statistique ou s'ils n'en sont que des formes primitives et incomplètes. Pour répondre à cette question, nous nous référons aux définitions que l'Encyclopédie de Diderot et d'Alembert donne des deux termes. Le compte est un état calculé ou non calculé d'effets possédés, administrés, acquis, reçus, dus ou dépensés ${ }^{18}$, tandis que l'état, en matière de compte, signifie un tableau ou mémoire, dans lequel on détaille la recette et dépense du comptable, ses reprises, etc. ${ }^{19}$. On constate que les notions de comptes et d'états s'entrecroisent. Elles n'impliquent pas de recours systématique au calcul et au tableau puisqu'elles se définissent également par rapport au texte et au mémoire. Cette ambivalence caractérise les premières formes de "statistiques" judiciaires sous la Révolution et doit nous rendre prudent quant à l'utilisation de la notion de "statistique ». Il nous appartiendra de définir, à partir de la pratique, non seulement le sens et l'objet des comptes et états mais aussi la place qu'ils occupèrent dans l'histoire de la statistique judiciaire.

\section{Les premiers comptes révolutionnaires}

5 Le premier document attestant la création d'une « statistique judiciaire » régulière date de l'époque de l'assemblée Législative. Il s'agit de la circulaire du 27 novembre 1791 par laquelle le ministre de la Justice, Duport-Dutertre, proclame le règne de la loi : la révolution est finie: la Constitution est faite. La loi est vraiment l'expression de la volonté générale; son exécution est le vou de la Nation et $d u R^{2 i^{20}}$. Ce sentiment d'accomplissement est compréhensible si l'on considère que la circulaire apparaît peu après la proclamation, en septembre 1791, de la première constitution révolutionnaire et qu'elle fait suite aux importantes réformes judiciaires entreprises au cours de la même année ${ }^{21}$. S'adressant aux juges, le ministre décrit la finalité de leurs fonctions : protéger la sûreté générale, faire respecter les lois qui la garantissent et réprimer les délits qui la troublent. Afin d'assurer plus d'exactitude dans l'administration de la Justice et de mettre sous les yeux de Sa Majesté, le tableau fidèle de vos travaux ${ }^{22}$, il propose que chaque tribunal lui envoie, à partir du premier janvier 1792, trimestriellement, l'état des causes jugées et ne doute pas que ce motif ne contribue à exciter votre zèle. En demandant de présenter au Roi un tableau de l'activité des tribunaux et d'y trouver, par conséquent, une source de motivation, le ministre confère aux états une fonction de contrôle des magistrats. Les indications concernant les activités à surveiller restent, cependant, encore floues. Elles mentionnent les opérations, les causes ou encore les travaux des tribunaux, sans préciser de quoi il ressort.

6 Nous n'avons retrouvé aucune trace de ces états. Il est donc impossible de vérifier s'ils ont été réalisés ou non. Mais l'intérêt de la circulaire de 1791 réside dans le fait qu'elle présente un certain nombre de préoccupations telles que le contrôle des magistrats, la dénonciation des infractions aux lois, la protection de la sûreté intérieure de la République et la présentation des états sous forme de tableaux, qui se rencontreront dans les autres «statistiques judiciaires" de la Révolution. En réalité, seule la périodicité trimestrielle constitue une originalité puisqu'elle ne survivra pas à l'an II. 
Elle s'avérait alors la mieux adaptée à la réalisation des comptes mais l'urgence de situation politique poussera le gouvernement à accélérer la fréquence du contrôle. Elle ne réapparaîtra qu'en avril $1806^{23}$.

\section{Les comptes de l'an II}

7 Si l'on excepte la circulaire de 1791, les principales traces attestant la création d'une « statistique judiciaire » régulière remontent à l'époque de la Terreur. Le 14 frimaire an II (4/12/1793), la Convention vote la création du gouvernement révolutionnaire. Les articles 7 et 10 de la section II $^{24}$ établissent l'envoi, par les tribunaux, de comptes décadaires et mensuels capables de relater l'activité générale (aussi bien judiciaire qu'administrative) de l'ensemble des juridictions. Jusque l'an III, l'application de cet arrêté ne laisse aucune trace. Il semble pourtant qu'il ait été exécuté comme en témoignent deux circulaires du ministre de la Justice Gohier, datées du 30 pluviôse (18/2/1794) et du 29 ventôse an II (19/3/1794). La circulaire du 29 ventôse est adressée aux juges des tribunaux civils et criminels, aux commissaires nationaux, aux accusateurs publics et aux juges de paix. Elle a pour objectif de répondre aux difficultés soulevées par le personnel judiciaire quant au contenu et à la forme des comptes décadaires et mensuels. La première partie de la réponse ministérielle se révèle particulièrement instructive par rapport aux normes fondant ces comptes :

Le compte décadaire consiste dans l'exposé des différentes applications que les tribunaux ont fait de la loi, ou, ce qui est la même chose, des différents jugements rendus pendant le cours de la décade. Cet exposé doit être sommaire et, cependant fournir le moyen de juger si les lois ont été bien appliquées. Il faut donc se rapprocher du mode indiqué par l'art. XV du titre V de la loi du 24 août 1790, sur l'organisation judiciaire, et en conséquence,

$91^{\circ}$. Énoncer les noms et qualités des parties.

$2^{\circ}$. Présenter l'objet de la demande au civil, ou le titre d'accusation au criminel.

$3^{\circ}$. Exposer succinctement les motifs qui ont déterminé la majorité des opinions.

$4^{\circ}$. Terminer l'analyse du jugement, dans laquelle on indiquera, sans les citer en entier, les articles des lois qui auront été appliqués.

10 Le compte de chaque mois doit, suivant l'article $\mathrm{X}$ de la section II, contenir l'analyse des délibérations et de la correspondance ${ }^{25}$.

11 Le compte décadaire occupe la majeure partie de l'exposé et pose manifestement le plus de difficultés quant à sa présentation et son contenu. Pour résoudre ces difficultés et proposer un modèle de référence aux tribunaux, Gohier se base sur l'article 15 titre $\mathrm{V}$ de la loi du 24 août 1790, qui établit les différentes parties des jugements de première instance et d'appel. Le compte décadaire doit présenter quatre types d'informations : identité du prévenu - nature de la prévention - jugement (accompagné des motifs du jugement) - analyse du jugement (accompagnée des articles de lois fondant le jugement). Le compte mensuel contient l'analyse des délibérations et de la correspondance des tribunaux. Il retrace, une nouvelle fois, l'activité de jugement des juridictions mais en y relatant, parallèlement, leur activité administrative. Après avoir collecté les différents comptes, le ministre de la Justice est chargé d'en présenter les résultats devant le Conseil exécutif. 
12 La seconde partie de la circulaire ministérielle précise l'objectif des deux comptes qui n'était pas clairement formulé par la loi du 14 frimaire an II :

Il n'est pas besoin, je pense, Citoyens, que j'insiste sur l'objet que la Convention s'est proposé en vous prescrivant l'obligation de rendre des comptes aussi fréquents; le titre seul de la loi du 14 frimaire vous l'indique assez. Toutes les parties d'un gouvernement révolutionnaire doivent être dans une continuelle activité, et se mouvoir dans un sens uniforme. Pour empêcher le mouvement de se ralentir et de changer de direction, une surveillance active est indispensable, et pour l'exercer utilement, en ce qui concerne l'ordre judiciaire, il faut connaître toutes les opérations des fonctionnaires publics, chargés de l'administration de la Justice. (...) $\mathrm{Au}$ surplus, c'est en méditant avec attention la loi du 14 frimaire, que vous parviendrez à résoudre vous-mêmes, Citoyens, les difficultés qui pourraient encore se présenter; c'est dans cette loi destinée à imprimer le mouvement aux autorités constituées, que vous trouverez la règle de votre conduite; vous y verrez la sévérité avec laquelle doivent être jugées les prévarications, même les simples négligences des fonctionnaires publics, la persévérance que vous devez mettre à suivre vos travaux sans interruption, et généralement ce que vous devez faire pour être des magistrats vraiment républicains et révolutionnaires ${ }^{26}$.

13 L'établissement des comptes judiciaires, décrété le 14 frimaire an II, est donc intimement lié à la Terreur. Dans le contexte d'un maintien absolu de l'unité d'action $\mathrm{du}$ gouvernement révolutionnaire, ils ont pour objectif de surveiller l'activité des fonctionnaires judiciaires. Le ministre Gohier menace ensuite de sanctions toute négligence dans la tenue des comptes.

Le 12 germinal an II (1/4/1794), une loi supprime le Conseil exécutif et le ministère de la Justice. Ils sont suppléés par un organe exécutif : la Commission des administrations civiles, police et tribunaux, dont les actes sont contrôlés par le Comité de Salut Public ${ }^{27}$. Ce remaniement de l'organisation gouvernementale est l'occasion pour la nouvelle Commission de perfectionner les comptes décadaires. Dans un arrêté daté du 29 prairial an II (17/6/1794) et adressé aux juges et aux commissaires nationaux de l'ensemble des juridictions, la Commission précise le modèle qu'elle entend donner aux comptes :

La forme de ces comptes doit être simple. Ceux des tribunaux annonceront, dans une partie, la réception, la consignation, la publication et l'affiche des Lois; dans l'autre, ils offriront l'analyse des Jugements rendus; cette analyse devra être très succincte, et néanmoins très claire; dans une troisième partie, les questions qui se seront présentées dans l'application des Lois, pour qu'elles soient transmises au Comité de Législation; dans une quatrième, les arrêtés ou délibérations d'ordre, de discipline. Enfin les observations générales. Les tribunaux de districts devront avoir un article séparé pour le jury d'accusation ${ }^{28}$.

Le modèle de prairial, comme celui du 29 ventôse, a pour objectif de retracer l'ensemble des activités des tribunaux. Mais, contrairement à la circulaire de ventôse, aucune référence n'est faite à la structure des comptes mensuels. Ce silence laisse supposer un traitement identique des deux comptes, sentiment qui sera confirmé par l'analyse des comptes de l'an III (voir chapitre IV). Le modèle de prairial témoigne d'une volonté de rationaliser la structure et le contenu des comptes. Les perfectionnements semblent avoir été efficaces puisque les directives de prairial resteront inchangées pendant un an et demi, jusqu'à la séparation de la Convention. L'avènement du Directoire apportera ensuite plusieurs modifications.

La fin de la Terreur n'entraîne donc pas la disparition des comptes judiciaires. Au contraire, la chute du gouvernement révolutionnaire donne l'occasion d'impulser un nouveau mouvement à l'envoi des comptes qui sont désormais sous la responsabilité du 
Comité de législation, suivant l'article 9 titre II de la loi du 7 fructidor an II $(24 / 8 / 1794)^{29}$. Par son arrêté du 22 fructidor an II (8/9/1794), le Comité de législation ordonne l'exécution de la loi du 14 frimaire aux administrations de département et de district, aux tribunaux civils, criminels et de police. Étant donné le changement des organes de gouvernement, le compte décadaire est désormais adressé à la Commission des administrations civiles, police et tribunaux et au Comité de législation, tandis que le compte mensuel est destiné uniquement au Comité. À partir des comptes mensuels, le bureau de l'exécution des lois et de la surveillance sur les corps administratifs et judiciaires du Comité établit des comptes globaux, synthétisant les informations fournies par les comptes des tribunaux. L'objectif des comptes est identique à celui qui prévalait sous la Terreur. Il s'agit de vérifier l'application des lois et de surveiller l'ensemble de l'activité des tribunaux.

\section{La tenue des comptes de l'an II face aux pouvoirs constitutionnels}

Par rapport à 1791, époque au cours de laquelle la responsabilité et la gestion des comptes incombait au ministère de la Justice, on assiste à un emprise progressive des organes législatifs sur l'ensemble des institutions judiciaires. Depuis la dissolution des ministères, le 12 germinal an II, la surveillance des autorités judiciaires est exercée par Commission des administrations civiles, de la police et des tribunaux, sous le contrôle du Comité de Salut Public. À la fin de la Terreur, l'implication du pouvoir législatif se maintient puisque la loi du 7 fructidor an II et l'arrêté du 22 fructidor an II attribuent explicitement au Comité de législation de la Convention la surveillance des tribunaux et la gestion des comptes. Cette fluctuation des compétences des corps constitués est révélatrice des changements de régime et de l'évolution des équilibres entre pouvoirs, qui tend à subordonner l'Exécutif au Législatif. La limitation des compétences du pouvoir exécutif apparaît également à travers le choix des fonctionnaires autorisés à tenir un compte. L'article 7 de la loi du 14 frimaire an II confiait l'application des lois civiles et criminelles aux tribunaux et leur réservait, en principe, la charge expresse d'en rendre compte tous les dix jours au conseil exécutif. Cependant, dans une circulaire du 30 pluviôse an II, adressée aux commissaires nationaux près les tribunaux de district et aux accusateurs publics, le ministre de la Justice, Gohier, donne une interprétation différente : quoique dans cet article, il ne soit pas fait mention directement des commissaires nationaux, le comité [de Salut Public] a pensé que, chargés d'exécuter et de faire exécuter la loi, vous deviez compte [sic] de son exécution, comme les tribunaux de son application et que faisant en quelque sorte partie de ces tribunaux, votre compte particulier, conformément à l'article 7, devait être rendu aux mêmes époques et à la même autorité, c'est-à-dire au Conseil exécutifín. Parallèlement à l'envoi des comptes des tribunaux, le ministre de la Justice demande donc aux commissaires nationaux l'envoi de comptes relatant l'exécution de la loi. La demande est révélatrice d'une instrumentalisation progressive des organes gouvernementaux par le Comité de Salut Public, afin de mieux contrôler les autorités judiciaires. Le ministre de la Justice exprime ouvertement cet objectif, en rappelant aux commissaires que leur travail porte $1^{\circ}$ sur l'exécution de la loi, quand c'est par vous-mêmes que vous l'exécutez. $2^{\circ}$ sur la surveillance de cette même exécution quand elle est confiée à d'autres officiers publics soumis à votre inspection. C'est surtout sur ce second point que je crois devoir appeler votre attention. (...) le nombre de dénonciations, suivant son augmentation ou diminution progressive, servira de thermomètre pour connaître si les lois sont bien ou mal exécutées dans toutes les parties de la République ${ }^{31}$. 
judiciaires doit disparaître. La rédaction de comptes est désormais réservée à l'institution judiciaire et les commissaires nationaux détachés auprès des différents tribunaux en sont exclus. L'exclusion est clairement exprimée par le Comité de législation aux commissaires nationaux près les tribunaux de district, le 9 brumaire an III :

Plusieurs d'entre vous, guidés par un zèle auquel on ne doit que des éloges, se chargent volontairement d'obligations que la loi ne leur impose pas. Ils se croient personnellement tenus d'adresser au comité de législation un compte général de leurs opérations, soit pendant le mois, soit pendant la décade, indépendamment des comptes auxquels les tribunaux sont assujettis. $\mathrm{Si}$, dans la loi du 14 frimaire, il est quelques dispositions qui vous soient applicables, ce sont celles contenues en l'article XVI de la section II. Comme fonctionnaires publics chargés personnellement de requérir l'exécution de la loi, ou la surveiller plus particulièrement, vous étiez tenus de correspondre exactement, tous les dix jours, avec le comité de salut public. Ce comité se trouvant dépouillé par la loi du 7 fructidor, de la surveillance sur les tribunaux, c'est avec le comité de législation, auquel elle est attribuée, que vous devez correspondre aujourd'hui; mais cette correspondance n'est point un compte. Les termes mêmes de l'article précité, vous indiquent que l'objet de cette correspondance, est de certifier les diligences faites pour l'exécution de chaque loi, de dénoncer les retards et les fonctionnaires publics négligents et prévaricateurs. (...). Vous devez donc, dans votre correspondance décadaire avec le comité de législation, vous borner à l'instruire de l'exécution ou de l'inexécution des lois : par là, vous serez débarrassés d'un travail superflu, et il vous restera plus de moments à donner aux fonctions importantes que vous remplissez ${ }^{32}$.

Le ton bienveillant mais ferme de l'arrêté tend à faire respecter scrupuleusement la loi du 14 frimaire an II. Il n'est plus question de « doubler » les comptes des tribunaux afin de les surveiller. La limitation des tâches des commissaires aux correspondances aura, comme nous le verrons au chapitre $\mathrm{V}$, pour conséquence tardive de priver le Directoire d'une législation autorisant explicitement ses commissaires à produire des comptes.

Il est difficile d'estimer si les tribunaux ont respecté la loi du 14 frimaire an II et les différents arrêtés et circulaires. Toutes les juridictions (civiles et pénales) étaient censées envoyer leurs comptes, mais seuls les comptes criminels nous sont parvenus ${ }^{33}$ pour une période s'étalant de vendémiaire an III jusque brumaire an IV.

\section{Les comptes des tribunaux criminels de l'an III}

21 À partir du mois de vendémiaire an III (septembre-octobre 1794), les présidents des tribunaux criminels envoient les premiers comptes mensuels au Comité de législation de la Convention. Ils répondent ainsi favorablement à l'arrêté du 22 fructidor an II. Il convient de relever la rapidité avec laquelle les tribunaux ont appliqué l'arrêté puisque au moins $77 \%^{34}$ d'entre eux s'y sont conformés dès vendémiaire. Les départements de la Nièvre et du Morbihan se montrent particulièrement zélés puisqu'ils réussissent à envoyer un compte pour le mois de fructidor an II. Il est toutefois malaisé d'évaluer l'assiduité des juridictions dans l'envoi de leurs comptes mensuels, étant donné l'état fragmentaire des archives conservées. Nous avons retrouvé $60 \%{ }^{35}$ des comptes mensuels de vendémiaire à floréal, soit pendant les huit premiers mois de l'an III. Si l'on considère que la plupart de ceux de frimaire ont bien été rédigés ${ }^{36}$, nous atteignons une estimation minimale de $71 \%{ }^{37}$ de comptes envoyés. L'envoi concerne les 90 
départements composant la République de l'an III. Seuls les comptes mensuels des deux départements corses (Liamone et Golo) et de la Gironde n'ont pas été retrouvés.

Les tribunaux ont donc globalement bien respecté l'arrêté du 22 fructidor. Cette assiduité n'exclut cependant pas les rappels à l'ordre comme en témoigne le formulaire que le Comité de législation imprima, le 8 pluviôse an III (27/1/1795), afin de réprimander individuellement les tribunaux négligents ${ }^{38}$. La mise en garde fut envoyée, le 22 pluviôse (10/2/1795), à tous les tribunaux qui ne s'étaient pas conformés, depuis quatre mois, à l'arrêté du 22 fructidor. Les réponses des tribunaux nous éclairent sur les causes des retards dans l'envoi des comptes mensuels. Ainsi, le greffier du tribunal criminel de l'Aisne se justifie par des motifs politiques. Il jure n'avoir jamais eu connaissance de l'arrêté du 22 fructidor an II et en rend responsable les président et accusateur public qui furent remplacés par le représentant Pérard. Le tribunal criminel des Hautes Pyrénées invoque, de son côté, l'installation tardive de l'accusateur public et du président qui ne prirent leurs fonctions que le 12 nivôse an III. Quant au tribunal criminel de la Drôme, si cette analyse n'a point été mise sous vos yeux, c'est sans doute parce qu'elle se sera égarée dans vos bureaux ${ }^{39}$. Mais, pour la majorité des tribunaux, la principale cause de la perte ou du retard des comptes est attribuée à la poste. La réponse formulée par les juges du Finistère est révélatrice de la dépendance des tribunaux à l'égard du service postal: nous ignorons par quelle fatalité ces comptes régulièrement mis à la poste à votre adresse ne sont pas parvenus jusqu'à vous. S'il n'eut s'agi que d'un seul envoi notre surprise aurait été moindre mais vous nous parlez des trois comptes de brumaire, frimaire et nivôse qui vous ont successivement manqué. Devons-nous attribuer la perte à l'infidélité de la poste ou aux événements malheureux qui peuvent être arrivés aux courriers. Mais dans l'un et l'autre cas comment nous mettre à l'abri du reproche de négligence que nous n'avons pas mérité et que nous ne mériterons jamais dans ce genre ${ }^{40}$. Cependant, les problèmes de communication du courrier sont peut-être moins imputables au service postal lui-même qu'au rythme mensuel des comptes. Ce dernier paraît trop rapide et inadapté aux capacités limitées de la poste, dans la mesure où la plupart des comptes « perdus " parviennent finalement à destination mais fortement en retard.

$\mathrm{Au}$ cours des quatre derniers mois de l'année, on ne relève plus que trente-cinq comptes (dont quinze en thermidor). Étant donné l'état de conservation des archives, il convient de se montrer prudent et de ne pas considérer ce faible nombre comme révélateur d'une baisse d'activité dans la rédaction des comptes. Les départements de la Nièvre et de Seine-et-Oise maintiennent d'ailleurs leurs envois jusqu'aux mois de vendémiaire et brumaire an IV. Une telle persistance témoigne du fait que l'arrêté du 22 fructidor an II et la loi du 14 frimaire an II sont toujours d'application respectivement un an et un an et demi après leur publication. Ils ne seront abrogés que suite à la séparation de la Convention (et par conséquent la disparition du Comité de législation), le 4 brumaire an IV, et à l'établissement du Directoire.

\subsection{Les comptes mensuels des tribunaux criminels}

24 La forme des comptes mensuels n'était théoriquement réglée par aucune circulaire. Celle du 29 ventôse an II se contentait de décrire leur contenu tandis que la circulaire du 29 prairial an II ne concernait que les comptes décadaires. À travers l'analyse des comptes de l'an III, il est toutefois apparu que les tribunaux appliquèrent le modèle de 
prairial aux comptes mensuels. Comme nous l'annoncions au chapitre III, le modèle ${ }^{41}$ se présente en cinq parties, sous la forme suivante :

Tableau 1 : Modèle des comptes mensuels des tribunaux criminels de l'an III

\begin{tabular}{|c|c|}
\hline & $\begin{array}{l}\text { Réception et Publication } \\
\text { des Lois }\end{array}$ \\
\hline & Jugements (I). \\
\hline \multirow[t]{4}{*}{$\begin{array}{l}\text { (I) Cet article doit être divisé en trois colonnes: } \\
\text { La première contiendra les noms, prénoms, qualités, professions et } \\
\text { demeures des prévenus et condamnés. } \\
\text { La seconde désignera la nature des délits. } \\
\text { La troisième, l'analyse des jugements rendus }\end{array}$} & \\
\hline & Questions. \\
\hline & Arrêtés ou Délibérations. \\
\hline & Observations générales. \\
\hline
\end{tabular}

Les Jugements comprennent les condamnations, acquittements, annulations, renvois et sursis prononcés par le tribunal. Les Questions ont trait aux référés posés par les juges soit à la Convention soit au Comité de législation, à propos de problèmes procéduraux ou de vides juridiques. Les Arrêtés reprennent principalement les nominations de greffier et les tirages au sort des jurés. Enfin, les Observations générales correspondent à une catégorie " divers ». Le modèle reprend donc l'ensemble de l'activité répressive et administrative d'un tribunal criminel. En pratique, les tribunaux appliquèrent le modèle sous les formes les plus variées mais qui révélèrent un certain nombre de constantes :

\section{Comptes tabulaires}

Les comptes se présentent soit sous la forme d'un tableau ( $68 \%)^{42}$, soit sous la forme d'un texte continu ( $32 \%$ ). La majorité des comptes tabulaires $(76 \%)^{43}$ sont manuscrits. Le formulaire pré-imprimé reste l'exception. La division en cinq parties n'a été que faiblement respectée. $39 \%$ des comptes rapportent la seule activité de jugement, $22 \%$ la complètent par l'annonce de la réception et de la publication des lois et $26 \%$ des comptes ajoutent, à ces deux types d'information, les différents arrêtés, questions et observations du tribunal ${ }^{44}$. Si l'on constate une distinction nette entre Lois et Jugements, les catégories Questions, Arrêtés et Observations sont, la plupart du temps, indifférenciées. Les comptes du tribunal criminel des Landes constituent, de ce point de vue, une exception puisqu'ils se divisent en six catégories : tirages au sort des jurés, réception et publication des lois, interrogatoires, arrêtés, jugements.

La division en trois colonnes de la partie Jugements a été globalement respectée. $73 \%{ }^{45}$ des tableaux se présentent sous cette forme. Si certains tribunaux, tels que ceux du Morbihan ou de l'Aube, composent des tableaux à dix ou douze colonnes, leur contenu 
est sensiblement le même que celui du modèle à trois colonnes. Il ne s'agit que de clarifier, en les scindant, la présentation de données similaires. En ce qui concerne l'intitulé des colonnes, l'utilisation exacte des termes juridiques pose manifestement problème. Les tableaux sont hésitants sur la qualification des individus à comptabiliser. S'agit-il de prévenus, de condamnés ou d'accusés ? Dans le doute, l'intitulé est laissé en blanc $^{46}$. Ces atermoiements se perçoivent à travers l'analyse comparée des comptes mais ils sont, somme toute, relatifs puisque la majorité des tribunaux conservent, tout au long de l'an III, les mêmes intitulés pour leur tableau.

\section{Comptes analytiques}

Les comptes de l'an III se caractérisent par une approche analytique et littéraire. Si l'on prend le cas des comptes tabulaires, ils contiennent, pour chaque colonne, une somme d'informations transcrites sous une forme textuelle. Ainsi, le tribunal criminel de Corrèze $^{47}$ :

Tableau 2 : Structure analytique du compte mensuel du tribunal criminel de Corrèze

\begin{tabular}{|c|c|c|}
\hline $\begin{array}{ll}\text { Noms } & \text { des } \\
\text { prévenus } & \end{array}$ & $\begin{array}{l}\text { Nature des } \\
\text { délits }\end{array}$ & Analyse des jugements rendus \\
\hline $\begin{array}{l}\text { André Vitrat, âgé } \\
\text { de quarante-cinq } \\
\text { ans, concierge de } \\
\text { la maison d'arrêt } \\
\text { du district } \\
\text { d'Userche, } \\
\text { habitant }\end{array}$ & \begin{tabular}{|l} 
Prévenu \\
d'avoir \\
favorisé \\
l'évasion de \\
douze détenus \\
dans ladite \\
maison d'arrêt
\end{tabular} & $\begin{array}{l}\text { Sur la déclaration du juré de jugement qui a émis son suffrage } \\
\text { en conformité de la loi du } 3 \text { messidor, portant qu'il est } \\
\text { constant qu'il s'est évadé douze détenus de la maison d'arrêt } \\
\text { d'Uzerche la nuit du } 10 \text { au } 11 \text { fructidor, que Vitrat était } \\
\text { préposé à leur garde mais qu'il n'a pas favorisé leur évasion, } \\
\text { qu'elle a été occasionnée par l'effet d'une force majeure et } \\
\text { imprévue et que ledit Vitrat a fait tout ce qu'il était en son } \\
\text { pouvoir pour l'empêcher; le tribunal l'acquitte de l'accusation } \\
\text { contre lui portée, et il a été mis en liberté. Jugement du } 15 \\
\text { brumaire, an III. }\end{array}$ \\
\hline
\end{tabular}

Le degré de précision des comptes varie fortement d'un tribunal à l'autre. On passe de la simple mention du jugement jusqu'à l'analyse précise de l'ensemble de la procédure.

\section{Comptes chronologiques}

Les comptes sont, en principe, structurés chronologiquement par affaires. Cependant, la plupart de ceux mentionnant la réception et la publication des lois ou les arrêtés introduisent un classement thématique. Ces deux matières sont alors clairement séparées des jugements et placées soit en début, soit en fin de compte. Elles sont ensuite elles-mêmes classées chronologiquement.

31 L'analyse des comptes mensuels des tribunaux criminels permet, dès à présent, de faire quelques constats intéressants. En dépit des difficultés de communication, des aléas du courrier postal, et des urgences des crises $^{48}$ de l'époque, on relève non seulement une rapidité d'exécution des comptes, de la part de l'ensemble des juridictions, mais surtout une continuité dans leur envoi, jusqu'à la fin de la Convention. D'autre part, les comptes ont une continuité dans leur présentation analytique, tabulaire et chronologique. Ces constantes sont toutefois entrecoupées par de nombreuses 
imprécisions et hésitations. L'utilisation exacte du vocabulaire juridique dans les intitulés des colonnes se fait attendre; dans certains tribunaux ${ }^{49}$, c'est la structure même - en tableau - qui est abandonnée, sans raison apparente, pour « régresser » vers une structure textuelle; enfin, du point de vue analytique, l'absence de directives précises quant à la nature des informations demandées laisse aux tribunaux la liberté de donner un contenu plus ou moins exhaustif de leur activité et rend, de ce fait, difficile l'analyse catégorielle et rapide des données. Certains tribunaux semblent d'ailleurs avoir pris conscience de cette difficulté et multiplient par eux-mêmes les colonnes des tableaux. Mais les faux-pas restent finalement limités. Le système d'envoi des comptes mensuels paraît relativement fonctionnel. Il sera repris et perfectionné par le Directoire afin d'étendre son contrôle sur le champ judiciaire.

\subsection{Les comptes généraux du Comité de législation}

32 Les informations recueillies par les comptes des tribunaux criminels sont synthétisées dans des comptes généraux. Cinq d'entres eux ${ }^{50}$ ont été conservés pour les mois de brumaire, frimaire, nivôse, pluviôse et ventôse an III. Les comptes de brumaire et frimaire ont une double présentation. La première partie des comptes apparaît sous une forme littéraire qui offre un aperçu chronologique de l'ensemble de l'activité de chaque tribunal (condamnés/acquittés, sursis, interrogatoires des accusés, tirage au sort des jurés, renvois, traduction à une maison d'arrêt,...). En fin de volume, le compte se modifie et se présente sous une forme tabulaire découpée en six colonnes, sans intitulés, mais dont voici les thématiques :

Tableau 3 : Structure des comptes généraux de l'an III

\begin{tabular}{|lr|l|l|l|l|l|}
\hline $\begin{array}{l}\mathrm{Nb} \text { total } \\
\text { condamnés } \\
\text { acquittés }\end{array}$ & $\begin{array}{l}\text { de } \\
\text { et }\end{array}$ & $\begin{array}{l}\text { Condamnés ou } \\
\text { acquittés }\end{array}$ & $\begin{array}{l}\mathrm{Nb} \text { de condamnés } \\
\text { ou acquittés }\end{array}$ & Peines & Observations & $\begin{array}{l}\text { Types de } \\
\text { délit }\end{array}$ \\
\hline
\end{tabular}

Parallèlement au changement de présentation, nous assistons à une modification du contenu des tableaux. Désormais, il ne s'agit plus d'exprimer fidèlement l'ensemble de l'activité des tribunaux mais uniquement leur activité répressive. Un fossé se creuse donc entre des comptes mensuels qui détaillent chaque occupation des juridictions et des comptes généraux qui n'en retiennent que le nombre de condamnés et d'acquittés.

La forme tabulaire reste limitée pour les mois de brumaire et de frimaire puisqu'elle ne concerne respectivement que $35 \%^{51}$ et $16 \%^{52}$ des départements repris dans les comptes généraux. Elle constitue, par contre, l'entièreté des tableaux de nivôse, pluviôse et ventôse. Il faut cependant se garder d'une interprétation "évolutionniste » de l'élaboration statistique puisque tous les comptes ont été rédigés à la même époque, aux alentours de ventôse. La variation de la structure des comptes semble plutôt provenir des différents essais de rédaction du compte. Ce sentiment est conforté par un doublon partiel du compte général de brumaire, perdu dans un autre fonds d'archives ${ }^{53}$. Les données présentées sous forme textuelle dans la première version du compte de brumaire y sont retranscrites mais, cette fois, sous une forme tabulaire. 


\section{Analyse des comptes généraux}

$\mathrm{Si}$, au premier abord, les comptes généraux semblent bien structurés, une analyse plus fine de leurs données révèle plusieurs incohérences. Contrairement aux comptes mensuels, de nombreux départements sont manquants. Le compte général de brumaire concerne 57 départements, celui de frimaire, 69 départements, celui de pluviôse, 73 départements, celui de nivôse, 70 départements et enfin celui de ventôse est partiel avec seulement 36 départements. Notons qu'un certain nombre de départements apparaissent plusieurs fois dans le même compte sans que nous puissions l'expliquer, même s'il arrive que l'auteur du compte général copie deux fois le même compte mensuel (Loire) ou qu'un tribunal envoie un compte séparé pour le jugement de crimes spécifiques (Lot-et-Garonne).

Lorsque l'on compare les données des comptes mensuels avec celles retranscrites dans les comptes généraux, on constate une absence de concordance pour plus de la moitié des tribunaux ${ }^{54}$. Cette incohérence a plusieurs causes. Il y a, tout d'abord, les erreurs d'inattention. L'auteur du compte général comptabilise, par exemple, sept acquittements pour la Charente inférieure alors même qu'il n'en a décrit que six. Il peut également "oublier" de noter une condamnation (Ariège) ou une accusation (Cher). Mais les divergences les plus importantes touchent au traitement des acquittements :

Tableau 4 : Compte général du tribunal criminel de l'Aube, en nivôse an III

\begin{tabular}{|l|l|l|l|l|l|l|}
\hline $\begin{array}{l}\text { Département } \\
\text { de l'Aube }\end{array}$ & $\begin{array}{l}\text { Nb total de } \\
\text { condamnés } \\
\text { et acquittés }\end{array}$ & $\begin{array}{l}\text { Condamnés } \\
\text { ou acquittés }\end{array}$ & $\begin{array}{l}\text { Nb de } \\
\text { condamnés } \\
\text { ou acquittés }\end{array}$ & Peines & Observations & $\begin{array}{l}\text { Types } \\
\text { de délit }\end{array}$ \\
\hline \hline & 4 & condamnés & 1 & $\begin{array}{l}4 \text { années de } \\
\text { fer }\end{array}$ & & \\
\hline \hline & & & 1 & $\begin{array}{l}2 \text { années de } \\
\text { prison }\end{array}$ & & meurtre \\
\hline & & & 1 & $\begin{array}{l}10 \text { années de } \\
\text { gêne }\end{array}$ & & \\
\hline & & acquittés & 4 & 1 & $\begin{array}{l}\text { Mort } \\
\text { non } \\
\text { convaincus } \\
\text { de vols }\end{array}$ & \\
\hline
\end{tabular}

Les comptes généraux groupent les acquittements et les condamnations séparément, même lorsqu'ils interviennent dans une même affaire. Le classement s'établit donc sur base de l'individu, en l'occurrence l'accusé, et non sur base de l'affaire, comme c'est le cas dans les comptes mensuels. Chaque condamnation est ensuite systématiquement détaillée, tandis que, les acquittements prononcés dans toutes les affaires sont, la plupart du temps, indifférenciés et groupés sur une ligne. Cette méthode de classement rend difficile toute tentative de reconstitution des comptes généraux à partir des 
comptes mensuels. Une telle tentative s'avère impossible lorsque, comme ce fut le cas pour l'Ariège, les accusés jugés en pluviôse sont comptabilisés dans le compte général de nivôse ou encore lorsque des données du compte général ne sont pas présentes dans le compte mensuel correspondant (Calvados, Alpes Maritimes).

Les «hésitations» entre compte littéraire et tabulaire, l'absence de plusieurs départements, la répétition inopinée de certains d'entre eux, le mode de classement des données recueillies dans les comptes mensuels et la rédaction simultanée des cinq comptes généraux compliquent fortement la compréhension de la genèse et de l'organisation des comptes généraux. Les nombreux oublis, erreurs et incohérences des comptes nous recommande l'extrême prudence dans leur utilisation. Il n'en reste pas moins que nous sommes manifestement en présence de la mise en œuvre d'un outil statistique. Celui-ci se caractérise par un mode d'enregistrement central des informations départementales. Les données font, ensuite, l'objet d'un tri puis d'un choix de comptage à deux niveaux. Un premier comptage répartit les prévenus en deux catégories: le nombre de condamnés par affaire et le nombre d'acquittés. Le second s'opère selon le nombre total mensuel de condamnés et d'acquittés de chaque tribunal.

La loi du 14 frimaire et l'arrêté du 22 fructidor de l'an II ordonnaient aux tribunaux de rendre compte au Comité de législation, de l'application des lois et d'y joindre l'analyse de leurs délibérations et de leur correspondance. L'objectif des comptes est clairement exprimé. Il s'agit de vérifier l'application des lois et de surveiller l'ensemble de l'activité des tribunaux. Les comptes généraux permettent de comprendre la manière dont le Comité envisage d'exercer ce contrôle. Alors que les comptes mensuels détaillent l'activité des tribunaux (annulations, renvois, sursis, nomination, référé au corps législatif, tirage des jurés...) et se structurent par affaires, les comptes généraux relèvent uniquement le nombre de condamnations/ acquittement et s'articulent autours d'accusés anonymes. Le Comité de législation concentre, par conséquent, son attention sur l'activité répressive du tribunal. À travers les comptes généraux, il s'offre les moyens d'évaluer la proportion d'acquittés et de condamnés, de connaître le type de peines appliquées ou les motifs d'acquittement (acquitté sur la question intentionnelle, sur cas de force majeur,...). Mais le contrôle n'est que partiel. Ainsi, l'absence de l'individualisation des acquittements et de la mention des annulations de jugements empêche toute appréciation du comportement du jury de jugement et de l'efficacité répressive des tribunaux, qui constituent deux enjeux majeurs de la période révolutionnaire. S'agit-il de lacunes volontaires ou est-ce le résultat des tâtonnements liés à la mise en place d'un outil statistique? Il n'est malheureusement pas possible de trancher.

\section{Le tournant du Directoire}

Le 4 brumaire an IV (26/10/1795), la Convention thermidorienne se sépare, peu après avoir voté la Constitution de l'an III, et fait place au Directoire. La nouvelle Constitution ordonne la création des arrondissements judiciaires. La réorganisation de l'architecture pénale provoque un changement dans le mode de contrôle des tribunaux. En effet, l'article 29 titre III de la loi du 14 vendémiaire an IV (6/10/1795) établit, dans chaque tribunal correctionnel d'arrondissement, un bureau de renseignements où sera tenu un registre alphabétique de tous les individus qui seront appelés au tribunal correctionnel ou au jury d'accusation, avec une notice sommaire de leur affaire et des suites qu'elles ont eues. 
Contrairement aux dispositions de l'arrêté du 22 fructidor an II, il n'est donc plus ordonné aux tribunaux d'envoyer, chaque décade, un compte relatant l'application des lois et, chaque mois, une analyse de leurs délibérations et de leur correspondance. La loi du 14 vendémiaire an IV étant uniquement une loi de nature organisationnelle, le législateur a seulement désiré que le tribunal dispose d'un registre alphabétique des prévenus pour sa correspondance personnelle et son organisation interne ${ }^{55}$.

41 L'absence de référence explicite à un envoi décadaire ou mensuel de comptes prive le Directoire exécutif des outils légaux nécessaires au contrôle de l'activité des tribunaux. Ce vide législatif est d'autant plus gênant que l'article 147 de la Constitution de l'an III réserve non seulement au Directoire exécutif la surveillance et l'exécution des lois dans les administrations et tribunaux mais il précise que la surveillance doit se faire par l'intermédiaire des commissaires du gouvernement. Il ne pouvait donc pas utiliser les registres aux renseignements des tribunaux comme source d'information. Pour combler cette lacune, le Directoire exécutif arrête, le 20 pluviôse an IV (9/2/1796), que les commissaires près des municipalités et les commissaires près les tribunaux correctionnels enverront respectivement aux commissaires près les administrations départementales et aux commissaires près les tribunaux civils et criminels tout ce qu'ils recueilleront de relatif à l'exécution des lois, à la sûreté publique et particulière, à tous les troubles, à tous les désordres qui peuvent exister, ou qui pourraient survenir dans leurs arrondissements respectifs ${ }^{56}$. Si le Directoire est bien en quête d'information, il ne parle pas encore de comptes et ne spécifie pas de quelle manière l'information doit être présentée aux autorités supérieures. Cependant, cet arrêté marque un tournant capital. Alors que depuis l'an III, le contrôle de l'exécution des lois était sous l'étroite surveillance du Comité de législation de la Convention auquel les tribunaux envoyaient directement des comptes mensuels, à partir de l'an IV et de l'application de la Constitution de l'an III, la surveillance des lois est monopolisée par le pouvoir exécutif, en l'occurrence le Directoire exécutif. Le retour en force du pouvoir exécutif laisserait penser que l'on revient à une situation comparable à celle constatée en 1791, lorsque le ministre de la Justice, Duport-Dutertre, réclamait des états aux juges. Il existe, cependant, une différence de taille. Les juges sont, désormais, exclus de l'élaboration des comptes. Celle-ci est réservée aux commissaires du gouvernement qui deviennent les seuls interlocuteurs du Directoire, en matière de contrôle de l'application des lois et de surveillance des tribunaux. L'exclusion des tribunaux s'explique par la stricte séparation des pouvoirs instituée par la Constitution de l'an III et qui caractérisera toute la période directoriale. Les juges se limiteront à l'administration de la Justice et les commissaires du gouvernement à son contrôle. Le contrôle ne concernera, cependant, pas la Justice civile puisqu'elle ne comporte pas de ministère public. À travers l'arrêté du 20 pluviôse an IV, le Directoire marque clairement une volonté de concentrer son énergie et son champ de surveillance sur les juridictions répressives. Il faudra, toutefois, attendre huit mois avant que les dispositions de l'arrêté fassent l'objet de toute l'attention du gouvernement. Ce dernier arrête, le 4 frimaire an $\mathrm{V}$ (24/11/1796), que les commissaires près les tribunaux de police et correctionnels rendront, tous les dix jours, les états des délits commis et des jugements rendus dans leur ressort. 


\section{Les états du Directoire} chaque juridiction mais selon un système hiérarchique: chaque commissaire près le tribunal de police (canton) envoie un état au commissaire près le tribunal correctionnel (arrondissement) qui y joint son propre état et transmet ensuite la synthèse au commissaire près les tribunaux civils et criminels (département). Ce dernier envoie, le premier de chaque mois, les renseignements récoltés au ministre de la Justice qui en fait rapport, le 15, au Directoire exécutif. Les états des jugements criminels n'accompagnaient pas ceux des autres juridictions. Ils faisaient l'objet d'une correspondance et d'une réglementation séparées (voir chapitre VII). Le système hiérarchique met donc un terme au double emploi qui existait, depuis l'an II, entre comptes décadaires et comptes mensuels (voir chapitre III), pour ne conserver qu'un seul état décadaire, collecté mensuellement ${ }^{57}$. En adoptant la hiérarchisation, le Directoire a non seulement voulu opérer une rationalisation des méthodes de collecte de l'information mais également améliorer la surveillance exercée sur l'activité des tribunaux et des commissaires du gouvernement.

Une deuxième différence par rapport à l'an II réside dans la tenue de deux types d'états. Les états du Directoire se composent, en effet, d'un état des jugements et d'un état des délits, rédigés sur deux feuilles séparées, dont l'objectif est expliqué par la circulaire du ministre de la Justice, Merlin de Douai, en date du 28 floréal an V (17/5/1797) :

Le Directoire exécutif, Citoyens, attache la plus grande importance à l'exécution de son arrêté du 4 frimaire dernier. Chargé par la Constitution de pourvoir à la sûreté intérieure de la République, il a besoin, pour atteindre ce but, d'avoir constamment sous les yeux le tableau de la situation des divers départements, et de suivre en quelque sorte l'action de la Justice dans toutes les circonstances qui peuvent menacer la sûreté des personnes ou des propriétés. Vous devez sentir combien vous le seconderez efficacement, en apportant une attention soutenue à ce qu'il n'échappe à sa sollicitude rien de ce qui peut porter atteinte à l'ordre social. Son intention ferme et persévérante est de réprimer toute violation de la loi et de réparer toute injustice, faites-vous un devoir religieux de remplir ses vues : dans la sphère où il vous a placé, sachez tout voir, et qu'il puisse voir tout par vos yeux ${ }^{58}$.

Afin de connaître les délits menaçant la sûreté intérieure de la République et de contrôler l'action de la Justice, le Directoire donne la priorité aux données pénales et laisse de côté celles relatives à l'organisation du tribunal. Nous rencontrons ici des préoccupations similaires à celles constatées lors de l'analyse des comptes généraux de l'an III mais qui n'étaient alors pas encore clairement formulées. En combinant l'envoi d'états des jugements et celui d'états des délits, le Directoire se dote d'un outil de contrôle performant que l'extrait de la circulaire ministérielle du 30 brumaire an VI (20/11/1797) permet de mesurer :

J'ai sous les yeux des tableaux formés par des commissaires près les tribunaux criminels, où, d'un côté, ils me certifient l'envoi exact des états que leur ont adressés la plupart des autres commissaires près les divers arrondissements du département; et de l'autre, ils m'attestent que pendant tout le cours du mois précédent, il ne s'est commis, dans toute l'étendue du département, aucun délit, et rendu aucun jugement correctionnel ou de simple police; cette assertion, dont la vérité rendrait superflue l'existence de toute espèce de tribunal de répression, est malheureusement trop contraire à la réalité, pour qu'il me soit permis d'y ajouter foi, quelle que soit la bonne opinion que mon imagination cherche à se former des commissaires qui me la transmettent. Je crois sincèrement à la vertu; mais il m'est 
difficile de la présumer assez généralement affermie dans le cœur d'une grande masse d'hommes disséminés sur un vaste territoire, pour que, pendant l'espace d'un mois, tout un département ait donné le rare exemple d'une observation rigoureuse des lois, et qu'il ne s'y soit, dans cet intervalle, rencontré aucun individu qui les ait enfreintes, même légèrement ${ }^{59}$.

La hiérarchisation de la collecte, l'exclusion de toutes données non pénales et la création d'états spécifiques aux délits témoignent d'une volonté du Directoire de rationaliser et de perfectionner le système des comptes de l'an II, afin de mieux évaluer toutes les violations de la loi et de mieux surveiller les fonctionnaires judiciaires qui ont la charge de les poursuivre. Ces objectifs se rencontrent également dans le modèle des états de jugements.

Tableau 5 : Modèle des états des jugements établi par la circulaire du 10 frimaire an $\mathrm{V}$

\begin{tabular}{|l|l|l|l|l|l|l|l|l|}
\hline $\begin{array}{l}\text { Noms des } \\
\text { tribunaux }\end{array}$ & $\begin{array}{l}\text { Date des } \\
\text { jugements }\end{array}$ & $\begin{array}{l}\text { Noms des } \\
\text { condamnés }\end{array}$ & $\begin{array}{l}\text { Peines } \\
\text { qu'ils ont } \\
\text { prononcées }\end{array}$ & $\begin{array}{l}\text { Y-a-t-il eu } \\
\text { recours à } \\
\text { cassation de } \\
\text { la part des } \\
\text { condamnés ? }\end{array}$ & exécutés? & & $\begin{array}{l}\text { S'ils ne le } \\
\text { sont pas, } \\
\text { quelles }\end{array}$ & \\
& & & & $\begin{array}{l}\text { diligences } \\
\text { a-t-il été } \\
\text { fait pour } \\
\text { leur } \\
\text { exécution? }\end{array}$ & \\
\hline
\end{tabular}

Par rapport au modèle de l'an II, celui du Directoire fait l'objet de plusieurs améliorations. Il se présente d'emblée sous la forme d'un tableau, divisé en catégories bien sériées permettant de récolter une information précise. Cette division permet de réduire la taille des réponses au renseignement demandé, sans toutefois éliminer l'approche analytique et littéraire. Les intitulés des colonnes nous autorisent à classer l'information qu'elles contiennent en deux catégories. Les quatre premières colonnes concernent le contrôle de l'activité de jugement du tribunal, tandis que les quatre dernières s'adressent aux commissaires du gouvernement, chargés de l'exécution des jugements. On constate donc que si les arrêtés, référés et autres matières administratives abordées dans les comptes de l'an II ont été exclus, le Directoire intègre, pour la première fois, dans un même état, l'activité de jugement des tribunaux et le contrôle des commissaires.

Tout au long du Directoire, la présentation des tableaux fait l'objet de plusieurs améliorations. Le premier modèle est envoyé aux commissaires de chaque juridiction par Merlin de Douai, le 10 frimaire an V (30/11/1796), afin de leur rendre plus facile le travail qui doit en résulter, et par là économiser les moments que vous devez à toutes les parties de vos fonctions ${ }^{60}$. Mais ce premier modèle comporte un défaut majeur. Il ne prévoit pas la mention du délit. Aussi, dès le 28 nivôse (17/1/1797), Merlin envoie aux commissaires près les tribunaux correctionnels et de police un modèle rectificatif : en rédigeant ces états chaque décade, il sera nécessaire que vous fassiez mention dans la première colonne, non seulement de la date, mais encore de la nature des délits, et que l'indication même de cette colonne porte ces mots : nature et date du délit. Il conviendra aussi que vous ajoutiez à l'état des jugements une colonne qui rappelle le délit et qui soit placée la première. L'indication que vous y mettrez, doit être également conçue en ces termes: nature et date du délit ${ }^{61}$. Une troisième modification est apportée, le 28 floréal an V (17/5/1797), par Merlin, sans que nous en 
connaissions la nature. Il joint à la circulaire adressée aux commissaires près les tribunaux criminels deux tableaux plus complets que ceux que vous avez déjà reçus et qui me paraissent devoir mieux remplir l'objet de l'arrêté $d u$ Directoire ${ }^{62}$. Les différents perfectionnements témoignent du souci du ministère de créer un modèle idéal, capable d'assurer l'effet des mesures prises par le gouvernement ${ }^{63}$.

L'application de l'arrêté du 4 frimaire an V s'est fait relativement rapidement. Le 10 frimaire, Merlin envoie les modèles aux commissaires et les premiers états retrouvés se rapportent déjà à la première décade de nivôse ${ }^{64}$. L'expérience accumulée depuis l'an II dans la confection de comptes explique sans doute cette rapidité. Après des débuts prometteurs, l'envoi des états fait l'objet de nombreux retards dont les origines sont comparables à ceux de l'an II. Le manque de locaux, l'absence de registres, le refus du greffier, l'absence de rémunération, les difficultés de communication, la distance séparant les différents cantons et les déficiences des messageries sont les motifs les plus souvent avancés pour expliquer la perturbation des envois ${ }^{65}$. D'autre part, la périodicité décadaire des états s'avère, une nouvelle fois, inadaptée au rythme de la pratique. En effet, beaucoup de cantons n'enregistrant aucun délit dans la décade, les commissaires ont tendance à ne pas envoyer leurs états vierges et compliquent ainsi la synthèse des données au niveau de l'arrondissement.

49 Les perturbations dans l'envoi des états sont également perceptibles, au niveau central, à travers la correspondance du ministre de la Justice. De l'an V à l'an IX, parallèlement à une multitude de courriers individuels, le ministre envoie au moins sept circulaires rappelant à l'ordre les commissaires. Le ton employé est le plus souvent modéré, oscillant entre les invitations fraternelles et les marques de mécontentement. La circulaire du 20 nivôse an VII (9/1/1799) adressée par Lambrechts aux commissaires près les tribunaux correctionnels est, à cet égard, révélatrice :

Je ne puis croire, citoyens, que la négligence qu'un grand nombre des commissaires du pouvoir exécutif près les administrations municipales de votre canton apportent dans l'exécution de l'arrêté du Directoire exécutif du 4 frimaire an 5 ne provienne pas de celle avec laquelle vous surveillez leurs opérations respectives. J'ai lieu de présumer que leur résistance à cet égard céderait bientôt à vos représentations fraternelles si, à chaque omission de leur part, vous les rappeliez à l'exercice de leurs devoirs au nom de la loi, de leur conscience et je puis ajouter de leur intérêt personnel. Je vous invite, citoyen, à reprendre avec eux l'habitude d'une correspondance qui les maintiennent dans la ligne de leurs obligations. En conséquence, vous voudrez bien faire connaître mon mécontentement aux commissaires du pouvoir exécutif près les administrations municipales des cantons de ... qui ne remplissent que très imparfaitement les vues du Directoire exécutif, en vous envoyant de temps à autre des certificats négatifs de délits ou jugements de police survenus dans leurs cantons pendant le cours de la décade précédente ${ }^{66}$.

Face à l'inertie de certains commissaires, le mécontentement du ministre de la Justice se transforme parfois en exaspération susceptible d'entraîner des mesures disciplinaires, comme l'illustre la lettre adressée, le 16 prairial an VII (4/6/1799), par Lambrechts à son collègue de l'Intérieur :

L'inutilité de mes efforts, mon cher collègue, pour ramener à l'exercice de leurs devoirs divers fonctionnaires du pouvoir exécutif près les administrations municipales des cantons de plusieurs départements de la République me force de les déférer à votre sévérité. Les fonctions qu'ils remplissent auprès des tribunaux de police de leurs cantons respectifs les plaçant à cet égard sous ma surveillance particulière, je n'ai rien négligé pour qu'ils entretiennent régulièrement la correspondance que leur prescrit l'arrêté du Directoire exécutif du 4 frimaire an 5 
dont l'exécution littérale est du plus grand intérêt pour le maintien de la tranquillité publique et la poursuite des crimes. Mais ils s'obstinent à mépriser mes invitations fraternelles ainsi que celles que leur adresse de ma part chacun des commissaires près les tribunaux correctionnels dans l'arrondissement duquel ils sont placés. Je vous invite, mon cher collègue, à faire usage de l'autorité directe que vous exercez. Je ne vous indiquerai que les plus négligents et ceux desquels je désespère d'obtenir, par aucun moyen, qu'ils se conforment aux obligations qui leur sont tracées par l'arrêté du Directoire exécutif du 4 frimaire an $5^{67}$.

51 Si la multiplicité des circulaires témoigne des dysfonctionnements du système des états décadaires, il convient de ne pas interpréter ces troubles comme un échec général. L'un des principaux objectifs assignés aux états - la mesure des délits - est sans doute loin d'avoir été atteint, compte tenu de l'imperfection de leur rédaction et des aléas de leur envoi, mais l'on peut se demander dans quelle mesure les circulaires de réprimande n'ont pas atteint le second objectif, à savoir le contrôle de l'activité des fonctionnaires judiciaires puisque l'absence ou la mauvaise tenue des états des commissaires du gouvernement signifiait, aux yeux du ministre de la Justice, un manque de zèle dans les poursuites ou dans le travail des commissaires. Quant aux tribunaux, leur activité de jugement semble, contre toute attente, avoir bel et bien été analysée par le ministère de la Justice. C'est ce que tendent à prouver plusieurs lettres dont celle que Lambrechts adresse, le 27 prairial an VI (15/6/1798), au commissaire près le tribunal correctionnel de l'arrondissement de Waremme (département de l'Ourthe) :

Le tribunal de police du canton de Waremme, Citoyen, dans le cours de germinal et floréal dernier a rendu deux jugements l'un contre Nicolas Mangeon, l'autre contre François Materne, qui condamnent chacun de ces deux particuliers à une amende plus forte que celle à laquelle la loi restreint le droit des tribunaux de police. C'est un abus de pouvoir dont il est de votre devoir d'empêcher le retour par la surveillance exacte que vous avez à exercer sur ces tribunaux. Je vous invite à prévenir le commissaire du Directoire exécutif près l'administration municipale du canton de l'obligation que lui impose son ministère de s'opposer à ce qu'il soit dorénavant prononcé de tels jugements et d'engager le tribunal de police de Waremme à se renfermer par la suite dans les bornes de ses attributions. Vous voudrez bien exiger de ce commissaire qu'il vous transmette avec précision la date des jugements et la nature des délits qui les ont nécessités. Rien de plus informe que les états décadaires qu'il paraît vous avoir envoyés jusqu'à présent. Il ne lui suffit pas de paraître déférer à l'arrêté du directoire exécutif du 4 frimaire an 5 par l'envoi d'un tableau insignifiant. Il doit encore en observer littéralement toutes les dispositions qui, d'ailleurs, sont toutes faciles à exécuter ${ }^{68}$.

\section{Les placards des jugements criminels}

52 Le système des états décadaires prévu par l'arrêté du 4 frimaire an V (24/11/1796) ne s'applique pas aux jugements criminels. Ceux-ci font l'objet d'une réglementation particulière. Le 2 pluviôse an V (21/1/1797), afin de donner la plus grande publicité (...) aux jugements rendus contre les auteurs des crimes ${ }^{69}$, le Directoire exécutif arrête que chaque tribunal criminel imprimera, à la fin de chaque mois, un état sommaire des jugements portant condamnation, soit sur déclaration du jury, soit sur appel des tribunaux correctionnels. L'état énoncera la date de jugement, les nom, prénom, domicile, âge et profession du condamné, la nature et le lieu du délit, la peine prononcée et l'article de la législation pénale qui l'aura motivé. Les états seront imprimés sous forme de placards à raison d'un pour chaque commune de moins de 5 000 habitants et d'un par tranche de 5000 habitants pour les communes les plus 
peuplées. Deux exemplaires supplémentaires seront, en outre, tirés. L'un sera déposé au greffe du tribunal criminel, l'autre sera envoyé au ministre de la Justice.

Par rapport aux états décadaires, les états des jugements criminels se différencient principalement par leur publicité. Les mêmes placards sont destinés à la fois au ministère de la Justice et à la population. Ce caractère public permet de mieux comprendre la raison pour laquelle les états criminels ne relèvent que les condamnés et n'abordent pas l'exécution de la peine. Le 27 brumaire an VI (17/11/1797), le Directoire prend un nouvel arrêté qui modifie légèrement celui du 2 pluviôse. Désormais, la responsabilité de l'impression n'incombe plus aux tribunaux mais aux commissaires du gouvernement; l'état comportera le signalement des condamnés; les jugements contre lesquels on se pourvoit en cassation ne seront pas indiqués et, enfin, un placard supplémentaire sera envoyé au ministre de la Justice.

54 Alors que les états décadaires sont abandonnés en l'an IX, le Consulat maintient la production des placards criminels, tout en corrigeant certains de leurs inconvénients. Dans une circulaire du 3 pluviôse an IX (23/1/1801), le ministre de la Justice Abrial demande aux commissaires de continuer l'envoi des états sommaires des jugements criminels et d'indiquer, en marge ou sur une note manuscrite, les pourvois en cassation. Parallèlement, Abrial demande d'adresser un état des affaires qu'il reste à juger. Il faudra, cependant, attendre la circulaire du 15 thermidor an IX (3/8/1801) pour qu'un état des jugements d'acquittement soit réclamé ${ }^{70}$.

55 La création par le Directoire de deux types d'états de jugements - de police et correctionnels d'une part, criminels d'autre part - peut paraître étonnant. D'un côté, nous avons un outil assez précis permettant de vérifier l'exécution des lois et de contrôler le zèle des commissaires du gouvernement, de l'autre côté nous avons des états assez sommaires, destinés au public et ne reprenant que les condamnés. La différence entre les deux états semble témoigner de l'importance donnée par le Directoire à la surveillance des juridictions inférieures. Celles-ci jugeaient, en effet, un certain nombre de délits aux enjeux politiques importants tels que les délits forestiers pour les tribunaux de police ou la fraude de marchandises anglaises et les délits religieux pour les tribunaux correctionnels. L'absence d'un tel outil de contrôle pour les tribunaux criminels indiquerait que le ministère de la Justice avait plus de facilités à apprécier leur activité.

L'abandon des états décadaires sous le Consulat répond sans doute à un motif économique et s'explique par la lourdeur du fonctionnement d'un tel système. La rédaction et l'analyse des états occasionnaient une dépense d'énergie et de temps considérable tant à Paris que dans les départements. Nous en avons pour preuve la correspondance volumineuse du ministère de la Justice et celle du substitut près le tribunal criminel de l'Ourthe, dont au moins $15 \%$ étaient consacrées aux seuls comptes décadaires ${ }^{11}$.

\section{Conclusion}

57 Alain Desrosières considère la période 1789-1815 comme essentielle pour l'élaboration et la formation des outils de la statistique française. Il divise cette période en trois étapes: De 1789 à 1795, sont conçus des recensements et des enquêtes particulières, qui n'aboutissent pas parce que lancés dans des situations d'urgence, de pénurie et de guerre, et par manque de l'infrastructure administrative adéquate. Puis de 1795 à 1806, sont organisées des 
enquêtes globales sur les nouveaux départements, dans un esprit comparable à celui de la statistique allemande. Enfin, de 1806 à 1815, sont mises en place des statistiques quantitatives régulières, notamment agricoles et industrielles ${ }^{72}$. Cette tripartition est présente dans toutes les histoires de la statistique. Elle implique une vision "évolutionniste» de la statistique liée aux principaux changements de régimes politiques: Révolution, Directoire-Consulat, Empire. La place réservée à l'épisode révolutionnaire, à l'exclusion du Directoire, n'est guère valorisée dans la mesure où les urgences des crises provoquent une succession incohérente de demandes d'informations par le centre, mal suivies et contrôlées et généralement sans suite ${ }^{73}$. Cependant, les résultats de notre étude bousculent quelque peu cette vision et permettent de réévaluer les résultats obtenus par les premiers essais de «statistiques » judiciaires de la Révolution.

Est-ce déjà de la statistique ou n'est-ce encore que des formes primitives et incomplètes de la discipline ? ${ }^{74}$, la question n'est pas tranchée en l'absence d'archives suffisamment complètes. Les comptes généraux de l'an III laissent, toutefois, penser qu'une statistique judiciaire existait, dès cette époque, au moins au niveau central. Enregistrées à Paris, les données départementales sont triées et comptées. Des séries chiffrées sont ensuite établies et, sans que nous puissions les interpréter totalement, dévoilent les objectifs de contrôle du gouvernement. Il est malheureusement impossible de déterminer si la technique était déjà rodée ou seulement au stade de l'essai.

59 En reconstituant la généalogie et la filiation des différents comptes, nous avons pu démontrer la continuité et la cohérence des comptes et des états, qui sont considérés comme un outil de gouvernement par les différents régimes depuis au moins l'an II. Cette continuité s'exprime également au niveau de la pratique des comptes et de leurs objectifs. Ainsi, la rapidité d'exécution des comptes, la régularité de leur envoi et la constance de leur présentation constituent de véritables performances. De telles performances sont évidemment entrecoupées par de nombreuses imprécisions et hésitations dont prennent conscience les gouvernements successifs. Aussi, à chaque remaniement de l'organisation gouvernementale apparaissent de nouvelles directives destinées à améliorer et à rationaliser la structure et la collecte des comptes. Le modèle tabulaire divisé en catégories sériées et bien définies du Directoire constitue, de ce point de vue, l'étape la plus aboutie.

Les objectifs attribués aux comptes sont établis dès 1791. Ils doivent permettre de contrôler l'activité des magistrats et les infractions aux lois. L'enjeu est donc d'importance. Il se reflète à travers la fluctuation des fonctionnaires autorisés à tenir un compte. Les fluctuations sont intimement liées à l'évolution politique et aux rapports de force entre pouvoirs législatifs, exécutifs et judiciaires. On passe ainsi des comptes rédigés par les juges, en 1791, à des états rédigés par les commissaires, sous le Directoire, tandis que, parallèlement, les comités législatifs (Comité de salut public, Comité de législation) et les organes exécutifs (ministère de la Justice, Commission des administrations civiles, police et tribunaux) se disputent la surveillance des autorités judiciaires. L'avènement du Directoire marque une séparation plus claire entre les pouvoirs et met en évidence une obsession de contrôle de la part du gouvernement. Ce dernier, à travers la hiérarchisation de la collecte des états, l'exclusion de toutes données non pénales et la création d'états spécifiques aux délits se donne les moyens de contrôler toutes les violations de la loi et de surveiller l'ensemble des fonctionnaires 
des juridictions répressives - les juges comme les commissaires - qui ont la charge de signaler les infractions, d'appliquer la loi ou de contrôler cette application.

61 La difficile question de la réussite ou tout au moins de l'aboutissement des comptes et états n'est pas encore résolue. Les comptes généraux de l'an III et la nombreuse correspondance disciplinaire du ministère de la Justice, sous le Directoire, semblent indiquer qu'ils n'ont pas été directement archivés mais qu'ils ont fait l'objet d'un traitement par le gouvernement central. L'avènement du Consulat mettra un terme à l'expérience des comptes et des états révolutionnaires, jugés trop coûteux. Il faudra alors attendre la dictature napoléonienne pour que l'État se donne les moyens nécessaires à la construction des différentes statistiques judiciaires capables de contrôler non seulement la magistrature mais également des pans de plus en plus larges de la société (statistique de la Justice civile, statistique des condamnés, statistique des faillites,...).

\section{BIBLIOGRAPHIE}

Aubusson de Cavarlay, B., Huré, M.-S., Pottier, M.-S., Les statistiques criminelles de 1831 à 1981 : la base DAVIDO, séries générales, Déviance et contrôle social, Paris, CESDIP, 1989.

Bourgin, G., Note sur la division civile du ministère de la Justice et les archives de la commission des administrations civiles, police et tribunaux, Annales historiques de la Révolution française, 1929, pp. 256-269.

Bourguet, M.N., Déchiffrer la France. La statistique départementale à l'époque napoléonienne, Paris, Éditions des archives contemporaines, 1989.

Chauvaud, Fr., Le juge, le tribun et le comptable. Histoire de l'organisation judiciaire entre les pouvoirs, les savoirs et les discours (1789-1930), Paris, Économica, 1995.

Desrosières, A., La politique des grands nombres. Histoire de la raison statistique, Paris, La Découverte, 1993.

Durand-Barthez, P., Histoire des structures du ministère de la Justice 1789-1945, Paris, PUF, 1973.

Encyclopédie ou dictionnaire raisonné des sciences, des arts et des métiers, Stuttgart, F. Frommann, 1966-1988, reprint.

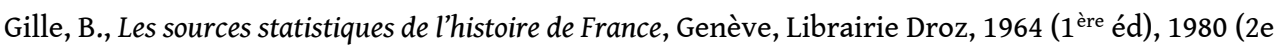
éd.).

Guéguan, I., Inventaire des enquêtes administratives et statistiques 1789-1795, Paris, Éditions du Comité des Travaux Historiques et Scientifiques, 1991.

Lascoumes, P., Poncela, P., Lenoël, P., Au nom de l'ordre. Une histoire politique du code pénal, Paris, Hachette, 1989.

Lecuir, J., Criminalité et moralité. Montyon, statisticien du Parlement de Paris, Revue d'Histoire Moderne et Contemporaine, juillet-septembre, tome XXI, 1974, pp. 445-493.

Le Nouveau Petit Robert, Paris, 1996. 
Leuwers, H., Un juriste en politique. Merlin de Douai (1754-1838), Arras, APU, 1996.

Leuwers, H., Rousseaux, X., La mise en place des nouvelles juridictions pénales dans la «Belgique » réunie (1795-1796), inInfluence du modèle judiciaire français en Europe sous la Révolution et l'Empire. Colloque organisé par le CHJL les 4, 5 et 6 juin 1998, L'Espace juridique, 1999, pp. 21-52.

Martucci, R., La Costituente e il problema penale in Francia (1789-1791), Milano, Giuffrè, 1984.

Perrot, J.C., L'âge d'or de la statistique régionale française (an IV-1804), Paris, Société des Études Robespierristes, 1977.

Perrot, J.C., Woolf, S.J., State and Statistics in France 1789-1815, Chur, Harwood academic publishers, 1984.

Perrot, M., Premières mesures des faits sociaux : les débuts de la statistique criminelle en France (1780-1830), Pour une histoire de la statistique, Paris, INSEE, 1977, pp. 125-137.

Recueil des circulaires, instructions et autres actes émanés du ministère de la Justice ou relatifs à ce département. An IV (1795-1796), Bruxelles, 1874.

Robert, Ph., Statistiques judiciaires, in Arnaud (A.J.), (Dir.), Dictionnaire encyclopédique de théorie et de sociologie du droit, Paris, L.G.D.J., 1993, pp. 582-583.

Rousseaux, X., «Que la Justice soit prompte, exacte et impartiale ». L'implantation de la Justice française dans le département de Sambre-et-Meuse (1795-1814), Annales de la Société archéologique de Namur, 1998, t. 72, pp. 97-145.

Rousseaux, X., Stevens, F., Tixhon, A., Les origines de la statistique pénale en Belgique (1795-1835), Déviance et société, 1998, 22, 2, pp. 127-153.

Tixhon, A., Le pouvoir des nombres. Une histoire de la production et de l'exploitation des statistiques judiciaires belges (1795-1870), Louvain-la-Neuve, thèse de doctorat inédite, 2001.

Woolf, S., Contribution à l'histoire des origines de la statistique : France, 1789-1815, in Bourget (M.N.) La statistique en France à l'époque napoléonienne, Bruxelles, Centre Guillaume Jacquemyns, 1981, pp. 45-125.

\section{ANNEXES}

\section{Chronologie de la statistique judiciaire révolutionnaire}

\begin{tabular}{|l|l|}
\hline $\begin{array}{l}\text { La Constituante } \\
\text { (17 juillet 1789- 30 } \\
\text { septembre 1791) }\end{array}$ & Néant \\
\hline $\begin{array}{l}\text { La Législative } \\
\left(\begin{array}{l}1^{\text {er }} \text { octobre 1791-20 } \\
\text { septembre 1792) }\end{array}\right.\end{array}$ & $\begin{array}{l}\text { 27 novembre 1791: circulaire du ministre de la Justice, Duport-Dutertre, } \\
\text { attestant, pour la première fois, la création d'états }\end{array}$ \\
\hline
\end{tabular}




\begin{tabular}{|l|l|}
\hline $\begin{array}{l}\text { La Convention } \\
\text { (21 septembre1792-4 } \\
\text { brumaire an IV) }\end{array}$ & $\begin{array}{l}14 \text { frimaire an II: loi établissant le gouvernement révolutionnaire et } \\
\text { l'envoi des comptes décadaires et mensuels de l'ensemble des } \\
\text { juridictions } \\
29 \text { ventôse an II: circulaire du ministre de la Justice, Gohier, établissant } \\
\text { le } 1^{\mathrm{er}} \text { modèle des comptes } \\
12 \text { germinal an II: loi supprimant les ministères et établissant la } \\
\text { Commission des administrations civiles, police et tribunaux } \\
29 \text { prairial an II: arrêté de la Commission établissant le 2ème modèle des } \\
\text { comptes } \\
10 \text { thermidor an II: fin de la Terreur } \\
7 \text { fructidor an II: élargissement des pouvoirs du Comité de législation } \\
22 \text { fructidor an II: arrêté du Comité de législation ordonnant l'exécution } \\
\text { de la loi du 14 frimaire an II en matière d'envoi des comptes }\end{array}$ \\
\hline $\begin{array}{l}\text { Le Directoire } \\
\text { 5 brumaire an IV-20 } \\
\text { brumaire an VIII) }\end{array}$ & $\begin{array}{l}14 \text { vendémiaire an IV: loi établissant un bureau de renseignement dans } \\
\text { chaque arrondissement judiciaire } \\
20 \text { pluviôse an IV: arrêté du Directoire exécutif réservant l'envoi des } \\
\text { états aux commissaires du gouvernement } \\
4 \text { frimaire an V: arrêté du Directoire exécutif établissant l'envoi des états } \\
\text { des délits commis et des jugements par les commissaires près les } \\
\text { tribunaux de police et correctionnels } \\
10 \text { frimaire an V: circulaire du ministre de la Justice, Merlin, établissant } \\
\text { le } 1^{\mathrm{er}} \text { modèle des états du Directoire. } \\
2 \text { pluviôse an V: arrêté du Directoire exécutif établissant l'envoi des } \\
\text { placards des jugements criminels }\end{array}$ \\
\hline
\end{tabular}

\section{NOTES}

2. Aubusson de Cavarlay, Huré, Pottier (1989); Desrosières (1993); Guéguan (1991); Gille (1964); Perrot, Woolf (1984); Perrot (1977); Rousseaux, Stevens, Tixhon (1998); Tixhon (2001).

3. Woolf (1981); Perrot (1977).

4. Perrot (1977, p. 21).

5. Bourguet (1989).

6. Woolf (1981, p. 52).

7. Bourguet (1989, p. 57); Woolf (1981, p. 52).

8. Perrot (1977, pp. 21-66); Woolf (1981, p. 58); Desrosières (1993, p. 44).

9. Guéguan (1991).

10. Rousseaux, Stevens, Tixhon (1998).

11. Guéguan (1991, p. 4).

12. Gille (1964, pp. 17-18).

13. Guéguan (1991, p. 6).

14. Gille (1964, p. 98).

15. Perrot (1977, pp. 125-126); Lecuir (1974).

16. Robert (1993, p. 582).

17. Le Nouveau Petit Robert (1996, p. 2142).

18. Encyclopédie ou dictionnaire raisonné des sciences, des arts et des métiers (1966-1988, vol. 3, p. 780).

19. Encyclopédie ou dictionnaire raisonné des sciences, des arts et des métiers (1966-1988, vol. 6, p. 28). 
20. AN (Archives nationales) BB2 21.

21. Martucci (1984); Lascoumes, Poncela, Lenoël (1989).

22. AN BB2 21.

23. Tixhon (2001, p. 42).

24. Art. 7 de la loi du 14 frimaire an II : (...) celle [l'application] des lois civiles et criminelles, [appartient] aux tribunaux, à la charge expresse d'en rendre compte tous les dix jours au conseil exécutif.Art. 10 de la loi du 14 frimaire an II : Tous les corps constitués enverront aussi, à la fin de chaque mois, l'analyse de leurs délibérations et de leur correspondance à l'autorité qui est spécialement chargée, par ce décret, de les surveiller immédiatement.

25. AN BB2 23.

26. AN BB2 23.

27. Bourgin (1929, p. 262).

28. AN BB2 24.

29. Art. 9 du titre II de la loi du 7 fructidor an II : Le comité de législation a la surveillance des administrations civiles et des tribunaux. Il est chargé des détails relatifs au recensement et à la classification des travaux commencés en exécution des décrets des 3 floréal et 11 prairial derniers. Il propose les lois relatives à sa partie, et prend, en se conformant à celles qui sont rendues, des mesures d'exécution relatives aux objets qui lui sont attribués.

30. AN BB2 23.

31. AN BB2 23.

32. AN DIII 320.

33. À l'exception d'un compte civil de vendémiaire an III, adressé par le tribunal du district de Calais (AN DIII 320).

34. 67 départements sur un total de 87 (AN DIII 325 à 328).

35. 418 comptes mensuels sur un total de 696 (AN DIII 325 à 328).

36. La quasi-absence, dans les archives, des comptes mensuels de frimaire (à peine 9 comptes nous sont parvenus) ne signifie pas qu'ils n'ont pas existé. Le compte général de frimaire montre, au contraire, que les comptes mensuels des tribunaux ont presque tous été envoyés (75 sur 87 comptes mensuels) mais qu'ils n'ont pas été conservés.

37. $493(418+75)$ comptes mensuels sur un total de 696 .

38. 8 pluviôse an III (date du bon pour l'impression de 600 exemplaires): Aux juges du tribunal... Par son arrêté du 22 fructidor qui vous a été transmis, le Comité avait déterminé que tous les tribunaux, tant civils que criminels, lui enverraient dans les premiers jours de chaque mois l'analyse des jugements qu'ils auraient rendus dans le cours du mois précédent. Le mois... s'est écoulé et le Comité n'a pas encore reçu la notice des jugements que vous avez rendus en... Ainsi, par une négligence qu'aucun motif ni prétexte ne peuvent légitimer ou excuser, vous avez contrevenu, non seulement à l'arrêté du Comité mais encore à l'article $1^{\text {er }}$ de la section $2^{\text {ème }}$ de la loi du 14 frimaire ( $2^{\text {ème }}$ année), sur lequel cet arrêté est fondé. Le Comité entend que vous lui transmettiez dans le plus court délai et séparément, l'analyse des jugements que vous avez rendus en... et successivement au commencement de chaque mois, celles des jugements que vous aurez rendus dans le mois précédent, et il vous prévient qu'en cas de retard, il sera obligé de provoquer contre vous les rigueurs de la loi (AN DIII 320).

39. AN DIII 326.

40. AN DIII 326 .

41. AN BB2 24

42. 59 comptes sur un total de 87 .

43. 45 tableaux sur un total de 59 .

44. Les $13 \%$ restants sont de formes hybrides.

45. 43 tableaux sur un total de 59 .

46. Un flottement similaire touche également la colonne nature des délits, où l'on tergiverse entre crime et délit. 
47. AN DIII 325.

48. Desrosières (1993, p. 54).

49. Tribunaux criminels de la Loire, du Haut-Rhin, du Gard, de la Marne et des Basses-Alpes.

50. Les cinq comptes généraux sont, à l'époque, dénommés comptes des tribunaux criminels ou dépouillements des comptes des tribunaux criminels. Nous les avons intitulés comptes généraux afin de les différencier des comptes mensuels des tribunaux criminels. Il convient de ne pas les confondre avec le Compte général de 1825. Précisons également que les auteurs ou rédacteurs des comptes généraux n'ont pu être identifiés, en l'absence de toute signature et autres marques sur les documents. Une identification n'est pas impossible mais elle sortirait du cadre de ce travail. Il faudrait, en effet, réaliser une étude de l'ensemble du personnel du ministère de la Justice. Une telle étude reste à faire, même si plusieurs travaux ont déjà abordé cette thématique (DurandBarthez (1973); Chauvaud (1995); Leuwers (1996).

51. 22 départements sur un total de 62 .

52. 13 départements sur un total de 80 .

53. AN BB2 97.

54. 14 tribunaux sur 23. Cette proportion a été obtenue à partir d'un sondage effectué auprès de 23 tribunaux criminels (Ardennes à Creuse) présents dans les comptes généraux et mensuels du mois de nivôse an III (AN DIII 325).

55. Nous avons gardé la trace de ces registres jusqu'en l'an XII.

56. Recueil (1874, p.161).

57. Rappelons, cependant, que depuis l'an III, la plupart des tribunaux ne différenciaient plus la présentation des comptes décadaires et des comptes mensuels (voir chapitre IV).

58. AN BB2 29.

59. AN BB2 8.

60. AN BB2 29.

61. AN BB2 29.

62. AN BB2 29.

63. AN BB2 29.

64. Archives de l'État à Namur, Tribunal de Namur, états du commissaire du pouvoir exécutif du tribunal correctionnel de l'arrondissement de St-Hubert (département de Sambre-et-Meuse).

65. Rousseaux (1998); Leuwers, Rousseaux (1999).

66. AN BB2 8.

67. AN BB2 8.

68. AN BB2 8.

69. AN AFIII* 160 .

70. Tixhon (2001, pp. 33-34).

71. Archives de l'État à Liège, Cours d'assise de Liège, registre de correspondances du substitut près le tribunal criminel du département de l'Ourthe, an VI.

72. Desrosières (1993, p. 44).

73. Ibidem, p. 54.

74. Tixhon (2001, p. 59). 


\section{RÉSUMÉS}

On assimile généralement la création de la statistique judiciaire à l'avènement de l'Empire napoléonien. Pourtant, les premières formes d'une telle statistique existent déjà sous la décennie révolutionnaire. Elles sont produites régulièrement, dans le but de surveiller l'activité des magistrats et les infractions aux lois. Compte tenu de l'importance de ces objectifs, les régimes révolutionnaires successifs veilleront à perfectionner les techniques d'enregistrement des statistiques judiciaires.

The creation of judicial statistics is normally associated with the advent of the Napoleonic Empire. Yet the practice of recording such statistics already existed during the Revolutionary decade. These statistics were collected regularly to check the work of magistrates as well as to record offences. Given the importance of these objectives, successive Revolutionary regimes continued to perfect the techniques of recording judicial statistics.

\section{AUTEUR}

\section{EMMANUEL BERGER}

Centre d'histoire du droit et de la Justice, UCL-Département d'histoire, Place Blaise Pascal, 1, B-1348 Louvain-la-Neuve, emmanuelberger@hotmail.com

Emmanuel Berger est aspirant du FNRS, chercheur au Centre d'histoire du droit et de la Justice, Université Catholique de Louvain, Belgique. Il prépare une thèse sur la Justice pénale sous le Directoire. Il a publié : De la Justice populaire à la Justice sécuritaire. L'audience au tribunal correctionnel de Bruxelles en 1795 et 2002, Les Cahiers de La Fonderie, 2003, 27, pp. 56-60; Le tribunal correctionnel de Bruxelles sous le Directoire (1795-1799), Annales de la Société Royale d'Archéologie de Bruxelles, 2003, 64, pp. 169-266; Le tribunal correctionnel de Bruxelles sous le Directoire, Bruxelles, Archives générales du Royaume, 2002. 